\title{
Evaluation of Diagnostic Value of SPECT/CT Imaging in Post-radioiodine Therapy in Thyroid Cancer
}

\author{
Asma Al Hatmi, "Anjali Jain, Alok K. Mittal, Samir Hussain
}

\begin{abstract}
ABSTRAC T: Objectives: This study aimed to investigate the value of single photon emission computed tomography/ computed tomography (SPECT/CT) imaging in well-differentiated thyroid cancer (DTC) after radioiodine (I-131) ablation/therapy for clinical staging and risk stratification. It also aimed to determine whether SPECT/CT would change the management plan or predict the clinical outcomes of DTC patients. Methods: A total of 78 DTC patients underwent first post radioiodine therapy "Whole body iodine-131 scintigraphy (WBS) along with SPECT/CT" at the Department of Radiology and Molecular imaging, Sultan Qaboos University Hospital, Muscat, Oman, between January 2014 and August 2017. Differences between WBS and SPECT/CT, change in clinical staging, risk stratification and management were recorded. The clinical outcome at 6-12 months was recorded. A generalised McNemar test was used to assess disagreement between WBS and SPECT/CT. Results: According to the American Thyroid Association (ATA) risk stratification, the sample showed low (35.8\%), intermediate (53.8\%) and high-risk groups (10.2\%) on WBS, which changed to $44.8 \%, 38.4 \%$ and $16.6 \%$, respectively, on SPECT/CT imaging. Overall change in risk stratification was noted in $16.7 \%$ and TNM stage in $11.5 \%$ of patients after SPECT/CT imaging. SPECT/CT changed the therapeutic plan and clinical outcome in 19.2\% of patients. Conclusion: SPECT/CT allows better detection and characterisation of metastatic lymph nodes and distant metastasis in DTC patients compared to WBS imaging alone. It alters TNM staging, ATA risk classification and management in a significant number of patients. It is recommended that SPECT/ CT should be done routinely along with WBS in well-differentiated thyroid carcinoma.
\end{abstract}

Keywords: Thyroid Cancer; Iodine; Ablation Techniques; SPECT/CT; Oman.

\section{AdVANCES IN KNOWLEDGE}

SPECT/CT has influenced American thyroid association risk stratification, TNM staging, management plan and clinical outcome in thyroid cancer patients.

\section{Application to Patient Care}

This study recommends that SPECT/CT should be an integral part of Whole body I-131 scintigraphy imaging protocol in differentiated thyroid cancer patients.

$\mathrm{T}$ HYROID CANCER HAS A GOOD PROGNOSIS AND is considered a treatable disease. The 5-year survival rates are $99.8 \%$ for localised disease, 97.0\% for regional metastases and $57.3 \%$ for distant metastases in well-differentiated thyroid cancer (DTC). ${ }^{1-3}$ It affects females more than males. The incidence of DTC is increasing globally over time due to the early detection of small lesions, which constitutes $87 \%$ of new cases. ${ }^{4,5}$ It is the second most common cancer among the Omani population as reported by the Ministry of Health in $2016 .{ }^{6}$ Treatment protocols of thyroid cancer patients vary depending on the initial American Thyroid Association (ATA) risk classification. ${ }^{2}$ ATA has divided thyroid cancer patients into three risk groups-low, intermediate and high risk. ${ }^{5}$ Radioactive iodine (RAI) treatment is an important aspect in the management of intermediate- and high-risk group patients. ${ }^{2}$ After total thyroidectomy, patients receive the first dose of radioactive iodine and routinely undergo post-therapy planar whole body scintigraphy (WBS) which detects residual thyroid tissue, lymph nodes and distant metastasis. ${ }^{3,4}$

The addition of single photon emission computed tomography/computed tomography (SPECT/CT) to planar WBS increases the diagnostic accuracy by improving the anatomic localisation of iodine uptake in the scan which changes the clinical stage and ATA risk classification. ${ }^{1,7}$ This further leads to alteration in the management plan such as the need for additional imaging, biopsy, surgery and external beam radiation or other systemic therapies. ${ }^{1,3}$

In addition to excellent three-dimensional localisation of abnormal iodine uptake, SPECT/CT helps in attenuation correction as well as detecting non-iodine avid distant metastases, which has a tremendous impact on the clinical outcome and rate of survival in DTC patients. ${ }^{3,8,9}$ Many institutions recommend I-131 SPECT/CT as part of the imaging protocol in selected high-risk patients with inconclusive findings on planar 
imaging. ${ }^{1-3}$ This study examined the importance of SPECT/CT with I-131 WBS in Omani patients, as there was no such similar study done locally.

\section{Methods}

This retrospective study was conducted at Sultan Qaboos University hospital, Muscat, Oman. A computerised search of medical records of the patient database was made from the hospital information system and Radiology information system (IntelliSpace PACS Enterprise, Philips Healthcare Informatics Inc., USA). A total of 183 patients who underwent postRAI (ablation/therapy) whole body scintigraphy were recorded from January 2014 to August 2017.

The inclusion criteria were: 1) Patients with histopathologically proven well-differentiated thyroid carcinoma who underwent first RAI therapy after total thyroidectomy; 2) Patients on whom both, WBS planar and SPECT/CT were performed on the same day; and 3) Availability of 6-12 months follow-up with diagnostic I-131 WBS. Five patients were excluded because they did not have well-differentiated thyroid cancers on histopathology and another 94 patients were excluded as SPECT/CT was not performed. Six patients were excluded because of the unavailability of 6-12 months follow up or diagnostic I-131 scan after the first therapy dose. Finally, a total of 78 patients were included in the current study [Figure 1].

After thyroidectomy, all patients received a high dose of radio-iodine for ablation/therapy. A diagnostic radioiodine scan was not performed as a protocol. Oral radioiodine dose was administered based on the surgical and pathological status of the tumour. Patients were classified into ATA low, intermediate or highrisk groups. A 1.1-3.7 GBq of RAI was administered to low- to intermediate-risk groups and $>3.7 \mathrm{GBq}$ was administered to high-risk group patients and patients with metastases as per departmental protocol. All patients were prepared with adequate thyroid hormone stimulation before the RAI therapy. The majority of patients (83.4\%) were kept off thyroxin for one month, while $16.6 \%$ of patients received recombinant thyroid-stimulating hormone (TSH) injection for thyroid hormone stimulation. A low iodine diet was recommended one week before RAI therapy.

Whole-body I-131 imaging was performed 3-7 days after the radioiodine dose on Symbia T-series dual-head SPECT gamma camera with multidetector CT scanner (Siemens Healthcare A/S, Denmark). Both WBS planar and SPECT/CT were acquired. Initially, anterior and posterior WB planar images were acquired at a speed of $12 \mathrm{~cm} /$ minute with high energy parallel hole collimator matrix of $256 \times 1,024$ and photopeak at $364 \mathrm{KeV}$ with a $15 \%$ window. Additional planar anterior images of the neck and lateral images of the abdomen were also acquired. At the same time, SPECT/CT images of the neck and chest were acquired from the skull base to the diaphragm. SPECT was acquired in step-and-shoot mode with 32 views and 180 degree rotation per detector. Acquisition time was between 30-60 seconds per step. Images were acquired with high energy parallel hole collimator with matrix of $128 \times 128$ and photopeak of $364 \mathrm{KeV}$ with a $15 \%$ window. CT images were acquired at $130 \mathrm{KeV}$, reference mAs 30 with $5 \mathrm{~mm}$ slice thickness. Axial, sagittal and coronal reconstruction of images was done on the Siemens workstation (Siemens Healthcare $\mathrm{A} / \mathrm{S})$.

Both WBS and SPECT/CT images were interpreted by a senior nuclear medicine physician, blinded to other laboratory and radiological image results. First, the planar images were interpreted. Any foci of uptake higher than the surrounding background (other than the physiological sites of uptake) were considered abnormal. Foci of abnormal iodine uptake in the medial portion of the lower neck, approximately in the thyroid bed, were categorised as positive for residual thyroid tissue.

An abnormal focus of uptake in the lateral part of the neck was considered as lymph node metastases. Foci of uptake that are very close to the medial part of the neck were equivocal for lymph node metastases. ${ }^{3}$ When more than one focus of uptake were seen in the medial part of the neck, the one with prominent uptake was considered as a residual tissue and the rest of the foci were interpreted as equivocal for lymph node metastases [Figure 2]. Foci of abnormal iodine uptake in mediastinum were categorised as mediastinal lymph node metastases, while abnormal foci adjacent to mediastinum were considered equivocal for lymph node metastases. Abnormal uptake in lung fields was considered as positive or equivocal for lung metastases. All areas of abnormal uptake beyond the neck and chest were also considered as positive or equivocal for metastatic foci and possible locations (bone, brain, liver, etc.) were also recorded. ${ }^{3}$

The findings were recorded as positive, negative and equivocal for thyroid bed, lymph node metastasis and distant metastasis. SPECT/CT images were also reviewed as a second step. Similarly, SPECT/ $\mathrm{CT}$ images were interpreted as positive and negative for thyroid bed, lymph node metastases and distant metastases. Non-iodine avid lesions seen on CT were also recorded. Changes observed in the interpretation of WBS planar and SPECT/CT were noted. Changes in ATA risk stratification and TNM staging were recorded. ${ }^{5,10}$ All patients were grouped into ATA low-, 


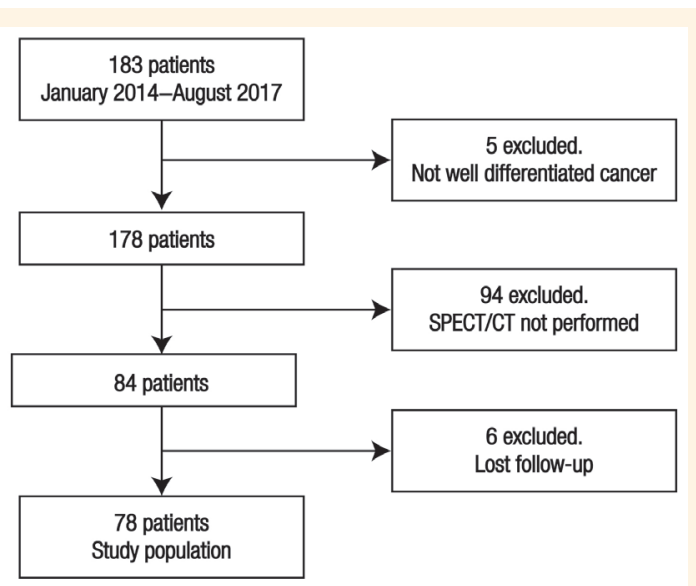

Figure 1: Flowchart showing the selection process used for patient enrolment.

intermediate- or high-risk groups and also categorised into four stages according to the $8^{\text {th }}$ edition of the American Joint Committee on Cancer (AJCC)/TNM staging of thyroid cancer. Changes in management plans like biopsies, additional cross-sectional imaging and further therapies were recorded. Clinical outcome like complete remission or additional treatment were noted on the 6-12 months follow-up.

A database in the SPSS programme, version 23 (IBM Corp., Armonk, New York, USA) was created for statistical analysis. By using the generalised McNemar test, disagreement between WBS planar and SPECT/ $\mathrm{CT}$ was assessed in three groups, including thyroid bed, lymph node metastases and distant metastasis. A $P$-value of $<0.05$ was considered statistically significant.

This study was approved by Sultan Qaboos University Hospital's Medical Research Ethical Committee and did not require informed consent (MREC\# 1567).

\section{Results}

Among the 78 patients included in our study, 64 (82\%) were females and 14 (18\%) were males. The age of the patients ranged from 7-76 years with a mean age of $41.6 \pm 16$. Papillary carcinoma was the most common histopathology presenting in $96 \%$ of patients compared to follicular and papillary carcinoma with poorly differentiated components in $3 \%$ and $1 \%$ of patients, respectively. These included nine patients of mixed papillary with follicular carcinoma, two patients with an oncocytic variant, one with a diffuse sclerosing type and one with Hurthle cell carcinoma. A background of multinodular goitre (MNG) was reported in 14\% of patients, 6\% had Graves' disease, 10\% had Hashimoto thyroiditis and 19\% lymphocytic thyroiditis. TSH levels for all patients during iodine administration were $>30 \mathrm{mIU} / \mathrm{L}$. Thyroglobulin (TG) levels ranged between 1-67,795 $\mu \mathrm{g} / \mathrm{L}$ and TG antibodies were positive in $20.5 \%$ of patients. Patients received high doses of radioactive iodine with activities ranging from 980-7,900 MBq.

There was total agreement between WBS planar and SPECT/CT image findings in residual thyroid tissue uptake. Out of 78 patients, 77 showed positive iodine uptake in thyroid bed on both, WBS planar and SPECT/CT images, suggesting residual thyroid tissue. Only one patient did not show any iodine uptake in the thyroid bed on both planar and SPECT/CT images.

There was a significant difference noted between the WBS planar and SPECT/CT images pertaining to lymph node metastases. On WBS planar imaging, 22 out of 78 patients were positive for lymph node metastases and 17 patients were equivocal for lymph node metastases. Out of the 22 patients (positive for lymph node metastases on WBS planar imaging), only 12 were positive on SPECT/CT images. In the remaining 10 patients (with false-positive lymph nodal uptake on WBS planar images), uptake was seen in thyroid bed on SPECT/CT images. These 10 patients showed complete remission at 6-12 months follow-up with no requirement for a second RAI dose. All equivocal lymph node metastases on WBS planar images were negative for lymph node metastases
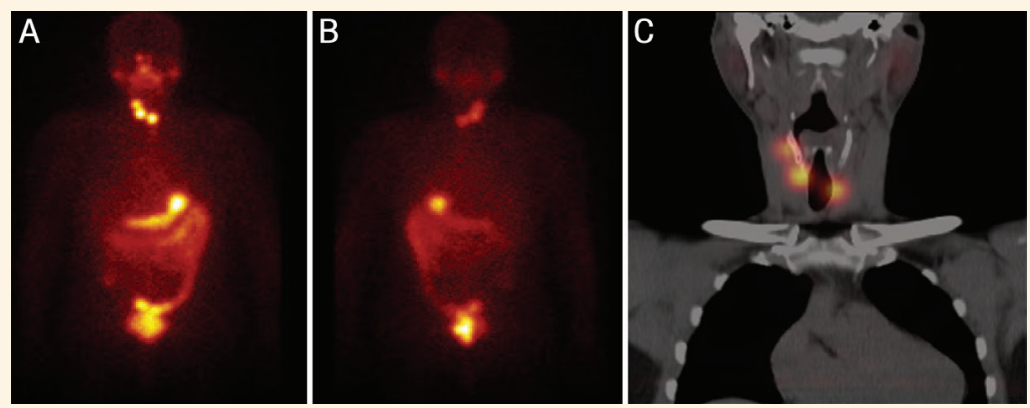

Figure 2: Thyroid bed versus lymph nodal metastases in a 76-year-old male patient. (A) Anterior and (B) posterior I-131 whole body planar images reveal multiple foci of iodine uptake in medial and right lateral neck region consistent with thyroid bed residual and neck lymphadenopathy. (C) Coronal SPECT/CT images of the neck showing iodine avid foci are localised in post-operative thyroid bed with no neck lymph nodal metastasis. 

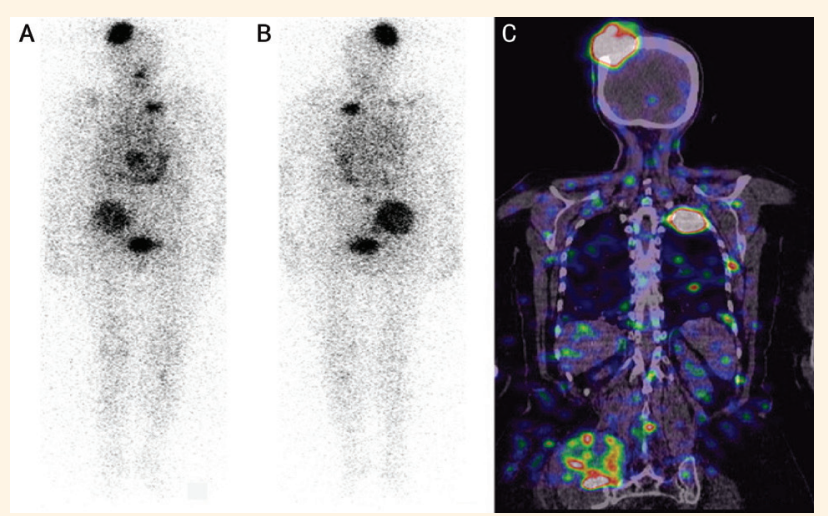

Figure 3: Iodine avid distant metastases in a 67-year-old female patient. (A) Anterior and (B) posterior I-131 whole body planar images reveal multiple areas of abnormal iodine avid distant metastases. (C) SPECT/CT coronal image showing iodine avid multiple lung, liver and skeleton distant metastases.

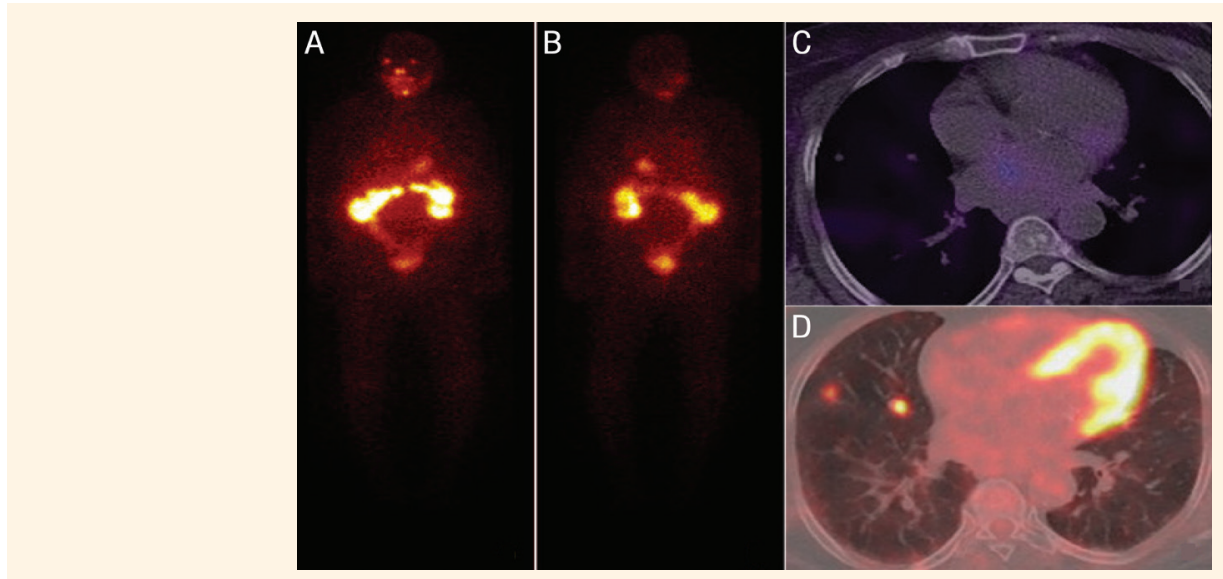

Figure 4: Non-iodine avid distant metastases in a 73-year-old male patient. (A) Anterior and (B) Posterior I-131 whole body planar images do not reveal any abnormal distant metastases. (C) SPECT/CT axial image showing non-avid multiple small pulmonary nodules. (D) 18-F FDG PET-CT axial fused image showing avid pulmonary nodules consistent with distant metastases.

on SPECT/CT imaging. The remaining 39 patients were negative for lymph node metastases on both WBS planar and SPECT/CT. There was a statistically significant difference between WBS planar and SPECT/CT imaging for lymph node metastases $(P$ $<0.01$ ) [Figure 2].

WBS planar imaging has shown distant metastases in five patients while two patients were equivocal for distant metastases. The most common site of metastases was the lung followed by bone and liver. SPECT/CT confirmed distant metastases in five patients, while two equivocal findings on WBS planar imaging turned out to be negative on SPECT/CT. There was a statistically significant difference between WBS and SPECT/CT imaging in distant metastases $(P$ $<0.01$ ) [Figure 3].

In addition, the CT portion of the SPECT/ CT also detected lung nodules in seven patients. These nodules did not show any iodine uptake on the post-therapy scan. Two of these patients showed complete resolution of nodules in the 6-12 months follow-up diagnostic I-131 WBS with normal TG levels $(<1 \mu \mathrm{g} / \mathrm{L})$, denoting these nodules were likely to be inflammatory. However, five patients in the 6-12 months follow-up showed persistent lung nodules with raised TG levels and were suspicious for metastases. Histopathologically, two patients showed papillary carcinoma with an oncocytic variant, one with poorly differentiated components, one follicular carcinoma and one papillary carcinoma. These patients were further evaluated by F-18 fluoro-deoxyglucose positron emission tomography/ computed tomography (FDG PET/CT) scan, which revealed abnormal increased FDG uptake and was considered as distant metastases of DTC. All of these patients were referred to an oncology centre and were treated with other systemic therapies (three patients) and radiotherapy (two patients) [Figure 4].

The difference between WBS planar imaging and SPECT/CT imaging categorising in the thyroid bed, neck lymph node and distant metastasis is well demonstrated in Figure 5.

Eight out of 10 patients with negative lymph node metastases on SPECT/CT imaging were restratified 

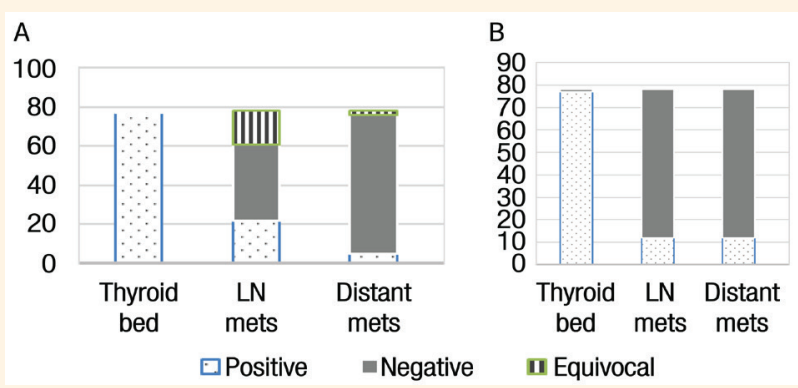

Figure 5: Bar graph showing a comparison of results between (A) WBS Planer imaging and (B) SPECT/CT imaging. There is no discrepancy in thyroid bed residual tissue, while there is a significant change in interpretation of neck lymph node and distant metastasis.

Table 1: Change in risk stratification after SPECT/CT

$\begin{array}{lcc}\begin{array}{l}\text { Thyroid cancer risk } \\ \text { stratification (\%) }\end{array} & \begin{array}{c}\text { Whole body } \\ \text { planar imaging }\end{array} & \text { SPECT/CT } \\ \text { Low } & 35.8 & 44.8 \\ \text { Intermediate } & 53.8 & 38.4 \\ \text { High } & 10.2 & 16.6\end{array}$

SPECT/CT = single photon emission computed tomography/computed tomography.

from the intermediate to the low-risk group. Risk stratification in two patients did not change due to the presence of extra thyroidal tumour extension. Five out of seven patients with non-iodine avid metastases were restratified to high-risk group.

ATA thyroid cancer risk stratification in these patients was reported as low- (35.8\%), intermediate(53.8\%) and high-risk groups (10.2\%) on planar imaging, which changed to $44.8 \%, 38.4 \%$ and $16.6 \%$, respectively, after SPECT/CT imaging. The overall change in risk stratification was noted in 13 (16.7\%) out of 78 patients [Table 1].

The percentage of change in TNM staging was smaller than the change in risk stratification. Out of 10 patients with negative lymph nodes on SPECT/ CT, TNM staging did not change in eight patients. These patients were $<55$ years old and continued to be categorised as stage I according to TNM staging for thyroid cancer. SPECT/CT down-staged the disease in two patients, as they were $>55$ years of age. The status of seven patients with non-iodine avid lesions changed from M0 to M1, thus upstaging the disease. The overall change in clinical staging was seen in nine (11.5\%) patients.

All patients showing change in TNM staging and risk classification were from intermediate- to high-risk groups.

Twelve patients with positive lymph nodes metastases on SPECT/CT imaging did not show remission at 6-12 months follow-up. These findings were correlated with neck USG and CT scans. Out of these 12 patients, one underwent re-surgery for complete lymph nodal dissection after confirmation of disease on fine needle aspiration cytology (FNAC). The rest of the patients underwent second RAI therapy. Ten false-positive lymph node metastases on WBS planar imaging (negative on SPECT/CT) showed complete remission on 6-12 months followup and did not require further RAI therapy, leading to change in clinical outcome. All five patients with non-iodine avid metastases on SPECT/CT (proven with F-18 FDG PET/CT) were upstaged from M0 to M1, which resulted in a change in the management plan. A total of 15 (19.2\%) patients showed changes in clinical outcome and management plan. Ten patients with negative lymph nodes went into remission on follow-up, while five patients with non-iodine avid metastases required further treatment in terms of systemic therapy and radiotherapy.

\section{Discussion}

SPECT/CT has become very popular in recent years. With the availability of hybrid cameras, it is being used frequently in nuclear medicine departments. Both functional and structural imaging can be performed at the same time, giving maximum benefit in terms of diagnosis and further management. SPECT/CT is also being used with WBS planar imaging in DTC resulting in substantial advantage for diagnosis and localisation of the disease. ${ }^{11-19}$

In the current study, all patients with DTC, who underwent both WBS planar and SPECT/CT imaging after the first RAI therapy were included. All the patients with only thyroid bed residual uptake showed no contradiction between WBS planar and SPECT/ CT findings. In some previous studies, SPECT/CT was acquired only in cases of diagnostic uncertainty and was not performed for clear thyroid bed residual uptake.,15 In the current study, all patients with residual thyroid bed tissue also underwent SPECT/CT and had complete remission after the first round of RAI therapy. Another study has shown similar results in thyroid bed uptake. ${ }^{3}$ 
Different studies in the literature have shown the advantage of SPECT/CT over WBS planar imaging in lymph node metastases, distant metastasis and in the management of DTC patients. ${ }^{20-25}$ In the current study, there was a disagreement in nodal metastases between WBS planar and SPECT/CT imaging $(P<0.01)$. These patients with false-positive nodes on WBS planar were downgraded on SPECT/CT images with complete remission of the disease at six months follow-up. On WBS planar imaging, these areas of iodine uptake were seen in the lateral or superior part of the neck and were interpreted as lymph node metastases, while they could be anatomically localised as residual thyroid tissue on three-dimensional tomographic acquisition of SPECT/CT images. Similarly, foci of uptake equivocal for lymph node metastases on WBS planar could also be delineated as residual thyroid tissue in the thyroid bed on SPECT/CT. In total, $34 \%$ of lymph nodal disease status was changed with SPECT/CT, which is similar to previously reported results in the literature. ${ }^{7,16}$ These studies changed and reclassified the lymph nodal status in $34-36.4 \%$ of patients with SPECT/CT imaging. However; these studies also interpreted lymph nodal disease from N0 status to N1 and from Nx status to N1. In the current study, lymph nodal status changed only from N1/Nx to N0. This could be explained either by differences in interpretation of findings on WBS planar with high suspicion of lymph nodal metastases. It may also be due to differences in surgical techniques and higher number of neck dissection during surgery in the current study, reducing the possibility of residual lymph nodal disease.

Findings with distant metastases have also shown significant disagreement between WBS planar and SPECT/CT imaging $(P<0.01)$. Two patients in the current study with equivocal finding of distant metastases were found negative on SPECT/CT. As shown by other studies, SPECT/CT reduced the number of indeterminate foci of metastases and also differentiated between benign physiological uptake from pathological uptake. ${ }^{3,18}$ In the current study, one of the foci in the mediastinum was localised to be oesophageal uptake. In another patient, diffuse linear uptake of iodine was seen in the lung, which was characterised as infective on CT images and showed resolution after a course of antibiotic. This is possible as the CT portion of SPECT/CT not only localises foci of uptake but also characterises the disease. Maruoka et al. have shown alteration of equivocal findings to benign findings in $38 \%$ of patients. ${ }^{3}$ The current study also showed a similar finding, although the number was much smaller. The detection of non-iodine avid lesions is very important in management since these patients cannot be treated with radioactive iodine and would need other treatment options. Diagnosis of these lesions on the post-therapy scan would save time and cost of additional imaging and would lead to proper management of the patient. Non-iodine lesions are also of prognostic significance and have a worse prognosis. In previous studies, authors have seen noniodine avid lesions in $21 \%$ of patients, while in the current study it was 9\%. ${ }^{2}$ According to the literature, dedifferentiated thyroid cancer can show noniodine avid metastasis in 30\% of cases and it is highly associated with certain aggressive histopathology like solid, insular and trabecular variants of poorly differentiated cancer, Hürthle cell carcinoma, papillary thyroid cancer with cribriform, columnar or tall cell variants. ${ }^{1}$

The current study showed a significant difference in WBS planar and SPECT /CT imaging in ATA risk stratification, TNM staging and management and thus the outcome of DTC. The current findings are similar to other studies where risk classification was changed in $6.4 \%, 25 \%$ and $35.6 \%$ of patients, respectively, after SPECT/CT and change in TNM stage was seen in $6.1 \%$, $25.9 \%$ and $21 \%$ of patients. ${ }^{2,3,16,20,26}$ Similarly, change in therapeutic plan was observed in $2.0 \%, 24 \%$ and $19.4 \%$ of patients after SPECT/CT. ${ }^{3,7,19}$ This variability in results may be explained due to differences in the qualitative assessment of image findings, patient population, age, histopathologies and variation in study protocols. Some studies have performed diagnostic I-131 scan before RAI therapy, while others did not. ${ }^{27,28}$ Other studies have performed SPECT/CT only in equivocal cases, while some studies did not include low-risk patients, as these patients were not treated with RAI. ${ }^{14,29}$ There were differences in study samples and type of analyses (lesion-based vs patientbased). ${ }^{2,19}$

Overall, additional SPECT/CT imaging improves diagnostic accuracy, anatomic localisation and helps in further characterisation of lesions, especially equivocal areas of iodine uptake and non-avid iodine lesions. This alters the TNM staging and initial risk stratification of the patients. The management plan also changes accordingly and it avoids additional imaging in most cases.

This study has limitations. It was a single-centre retrospective study with a small sample size and short term follow-up. Secondly, metastases were diagnosed on thyroglobulin levels and imaging results, while histopathology was not always available. Another limitation in this study was interpretation of WBS planar images with multiple foci of uptake in neck, where one prominent uptake was considered residual thyroid tissue and the rest of them as lymph node 
metastases due to limitation of planar images to localise the area of uptake. It has been seen on SPECT/ $\mathrm{CT}$ images that residual thyroid tissue can be seen as multiple foci of uptake.

\section{Conclusion}

SPECT/CT is a powerful diagnostic tool that allows better detection and further characterisation of lymph node and distant metastasis of well-differentiated thyroid carcinoma compared to WBS planar imaging alone. It also alters risk stratification, clinical staging and management plan of patients. It is recommended that SPECT/CT should be routinely performed in the evaluation of well-differentiated thyroid carcinoma patients after radioiodine therapy.

\section{CONFLICT OF INTEREST}

The authors declare no conflicts of interest.

\section{FUNDING}

No funding was received for this study.

\section{AUTHORS' CONTRIBUTION}

AJ and SH conceptualised the work. AJ supervised the work. AAH prepared the proposal and collected the data. AAH and $\mathrm{AJ}$ analysed and interpreted the data. AAH drafted the manuscript. AJ and AKM reviewed and edited the drafted manuscript. AKM collected, labelled and formatted the images. All authors approved the final version of the work.

\section{References}

1. Anca MA. Radioiodine scintigraphy with SPECT/CT: An important diagnostic tool for thyroid cancer staging and risk stratification. J Nucl Med Technol 2014; 42:170-80. https://doi. org/10.2967/jnumed.111.104133.

2. Grewal RK, Tuttle RM, Fox J, Borkar S, Chou JF, Gonen M, et al The effect of posttherapy 131I SPECT/CT on risk classification and management of patients with differentiated thyroid cancer. J Nucl Med. 2010; 51:1361-7. https://doi.org/10.2967/ jnumed.110.075960.

3. Maruoka Y, Abe K, Baba S, Isoda T, Sawamoto H, Tanabe Y, et al. Incremental diagnostic value of SPECT/CT with 131-I scintigraphy after radioiodine therapy in patients with welldifferentiated thyroid carcinoma. Radiology 2012; 265:902-9. https://doi.org/10.1148/radiol.12112108.

4. Avram AM, Fig LM, Frey KA, Gross MD, Wong KK. Preablation I131 scans with SPECT/CT in postoperative thyroid cancer patients: what is the impact on staging? J Clin Endocrinol Metab 2013; 98:1163-71. https://doi.org/10.1210/jc.2012-3630.

5. Haugen BR, Alexander EK, Bible KC, Doherty GM, Mandel SJ, Nikiforov YE, et al. 2015 American thyroid association management guidelines for adult patients with thyroid nodules and differentiated thyroid cancer. Thyroid 2016; 26:1-133. https:// doi.org/10.1089/thy.2015.0020.
6. Ministry of Health. Cancer incidence in Oman 2016. From: https://www.moh.gov.om/documents/272928/1232802/Can cer+Incidence+In+Oman+2016/3ff52524-8983-6088-147bfd09f7f262b4 Accessed: Nov 2020.

7. Kohlfuerst S, Igerc I, Lobnig M, Gallowitsch HJ, Gomez-Segovia I, Matschnig S, et al. Post therapeutic (131)I SPECT/CT offers high diagnostic accuracy when the findings on conventional planar imaging are inconclusive and allow a tailored patient treatment regimen. Eur J Nucl Med Mol Imaging 2009; 36:886-93. https://doi.org/10.1007/s00259-008-1044-2.

8. Durante C, Haddy N, Baudin E, Leboulleux S, Hartl D, Travagli JP, et al. Longterm outcome of 444 patients with distant metastases from papillary and follicular thyroid carcinoma: benefits and limits of radioiodine therapy. J Clin Endocrinol Metab 2006; 91:2892-9. https://doi.org/10.1210/jc.2005-2838.

9. Bybel B, Brunken RC, DiFilippo FP, Neumann DR, Wu G, Cerqueira MD. SPECT/CT imaging: Clinical utility of an emerging technology. Radiographics 2008; 28:1097-113. https://doi.org/10.1 148/rg.284075203.

10. Tuttle RM, Haugen B, Perrier ND. Updated American Joint Committee on Cancer/Tumor-Node-Metastasis Staging System for Differentiated and Anaplastic Thyroid Cancer (Eighth Edition): What Changed and Why? Thyroid 2017; 27:751-6. https://doi.org/10.1089/thy.2017.0102.

11. Yamamoto Y, Nishiyama Y, Monden T, Matsumura Y, Satoh K, Ohkawa M. Clinical usefulness of fusion of 131I SPECT and CT images in patients with differentiated thyroid carcinoma. J Nucl Med 2003; 44:1905-10.

12. Ruf J, Lehmkuhl L, Bertram $\mathrm{H}$, Sandrock D, Amthauer $\mathrm{H}$, Humplik B et al. Impact of SPECT and integrated low-dose CT after radioiodine therapy on the management of patients with thyroid carcinoma. Nucl Med Commun 2004; 25:1177-82. https://doi.org/10.1097/00006231-200412000-00004.

13. Tharp K, Israel O, Hausmann J, Bettman L, Martin WH, Daitzchman $M$ et al. Impact of 131I-SPECT/CT images obtained with an integrated system in the follow-up of patients with thyroid carcinoma. Eur I Nucl Med Mol Imaging 2004; 31:1435-42. https://doi.org/10.1007/s00259-004-1565-2.

14. Chen L, Luo Q, Shen Y, Yu Y, Yuan Z, Lu H, et al. Incremental value of 131I SPECT/CT in the management of patients with differential thyroid carcinoma. J Nucl Med 2008; 49:1952-7. https://doi.org/10.2967/jnumed.108.052399.

15. Wong KK, Zarzhevsky N, Cahill JM, Frey KA, Avram AM. Incremental value of diagnostic 131I SPECT/CT fusion imaging in the evaluation of differentiated thyroid carcinoma. AJR Am J Roentgenol 2008; 191:1785-94. https://doi.org/10.2214/AJR.0 8.1218 .

16. Schmidt D, Szikszai A, Linke R, Bautz W, Kuwert T. Impact of 131I SPECT/spiral CT on nodal staging of differentiated thyroid carcinoma at the first radioablation. J Nucl Med 2009; 50:18-23. https://doi.org/10.2967/jnumed.108.052746.

17. Wang H, Fu HL, Li JN, Zou RJ, Gu ZH, Wu JC. The role of single-photon emission computed tomography/computed tomography for precise localization of metastases in patients with differentiated thyroid cancer. Clin Imaging 2009; 33:49-54. https://doi.org/10.1016/j.clinimag.2008.06.024.

18. Aide N, Heutte N, Rame JP, Rousseau E, Loiseau C, HenryAmar $\mathrm{M}$, et al. Clinical relevance of single-photon emission computed tomography/computed tomography of the neck and thorax in postablation 131I scintigraphy for thyroid cancer. J Clin Endocrinol Metab 2009; 94:2075-84. https://doi. org/10.1210/jc.2008-2313.

19. Spanu A, Solinas ME, Chessa F, Sanna D, Nuvoli S, Madeddu G. 131I SPECT/CT in the follow-up of differentiated thyroid carcinoma: incremental value versus planar imaging. J Nucl Med 2009; 50:184-90. https://doi.org/10.2967/jnumed.108.056572. 
20. Wong KK, Sisson JC, Koral KF, Frey KA, Avram AM. Staging of differentiated thyroid carcinoma using diagnostic 131I SPECT/CT. AJR Am J Roentgenol 2010; 195:730-6. https://doi. org/10.2214/AJR.09.3458.

21. Barwick T, Murray I, Megadmi H, Drake WM, Plowman PN, Akker SA, et al. Single photon emission computed tomography (SPECT)/ computed tomography using Iodine-123 in patients with differentiated thyroid cancer: Additional value over whole body planar imaging and SPECT. Eur J Endocrinol 2010; 162:1131-9. https://doi.org/10.1530/EJE-09-1023.

22. Xue YL, Qiu ZL, Song HJ, Luo QY. Value of 131 I SPECT/CT for the evaluation of differentiated thyroid cancer: a systematic review of the literature. Eur J Nucl Med Mol Imaging 2013; 40:768-78. https://doi.org/10.1007/ s00259-012-2310-x.

23. Avram AM, Esfandiari NH, Wong KK. Preablation 131-I scans with SPECT/CT contribute to thyroid cancer risk stratification and 131-I therapy planning. J Clin Endocrinol Metab 2015; 100:1895-902. https://doi.org/10.1210/ jc.2014-4043.

24. Shen CT, Wei WJ, Qiu ZL, Song HJ, Luo QY. Value of posttherapeutic (131)I scintigraphy in stimulated serum thyroglobulin-negative patients with metastatic differentiated thyroid carcinoma. Endocrine 2016; 51:283-90. https://doi.org/10.10 07/s12020-015-0660-4
25. Wong KK, Zarzhevsky N, Cahill JM, Frey KA, Avram AM. Hybrid SPECT/CT and PET-CT imaging of differentiated thyroid carcinoma. Br J Radiol 2009; 82:860-76. https://doi. org/10.1259/bjr/25645894.

26. Szujo S, Sira L, Bajnok L, Bodis B, Gyory F, Nemes O, et al. The impact of post-radioiodine therapy SPECT/CT on early risk stratification in differentiated thyroid cancer; a bi-institutional study. Oncotarget 2017; 8:79825-34. https://doi.org/10.18632/ oncotarget.19781.

27. Ciarallo A, Rivera J. Radioactive iodine therapy in differentiated thyroid cancer: 2020 update. AJR Am J Roentgenol 2020; 215:285-291. https://doi.org/10.2214/AJR.19.22626.

28. Ahmed N, Niyaz K, Borakati A, Marafi F, Birk R, Usmani S. Hybrid SPECT/CT imaging in the management of differentiated thyroid carcinoma. Asian Pac J Cancer Prev 2018; 19:303-8. https://doi.org/10.22034/APJCP.2018.19.2.303.

29. Lamartina L, Leboulleux S, Terroir M, Hartl D, Schlumberger M. An update on the management of low-risk differentiated thyroid cancer. Endocr Relat Cancer 2019; 26:R597-610. https://doi. org/10.1530/ERC-19-0294. 